\title{
Operational Tau approximation for a general class of fractional integro-differential equations
}

\author{
S. KARIMI VANANI and A. AMINATAEI \\ Department of Mathematics, K.N. Toosi University of Technology, \\ P.O. Box: 16315-1618, Tehran, Iran \\ E-mails: solatkarimi@dena.kntu.ac.ir / ataei@kntu.ac.ir
}

\begin{abstract}
In this work, an extension of the algebraic formulation of the operational Tau method (OTM) for the numerical solution of the linear and nonlinear fractional integro-differential equations (FIDEs) is proposed. The main idea behind the OTM is to convert the fractional differential and integral parts of the desired FIDE to some operational matrices. Then the FIDE reduces to a set of algebraic equations. We demonstrate the Tau matrix representation for solving FIDEs based on arbitrary orthogonal polynomials. Some advantages of using the method, error estimation and computer algorithm are also presented. Illustrative linear and nonlinear experiments are included to show the validity and applicability of the presented method.
\end{abstract}

Mathematical subject classification: 65M70, 34A25, 26A33, 47Gxx.

Key words: spectral methods, operational Tau method, fractional integro-differential equations, error estimation, computer algorithm of the method.

\section{Introduction}

The main object of this work is to solve the fractional integro-differential equation of the following form:

$$
\begin{array}{r}
D^{\alpha} u(x)=F(x, u(x))+\int_{0}^{x} G(x, t, u(t)) d t, \\
m-1<\alpha \leq m, \quad m \in \mathbb{N}, \quad x>0, \\
B_{j} u(x)=\lambda_{j}, \quad \lambda_{j} \in \mathbb{R}, \quad j=0,1,2, \ldots, m-1,
\end{array}
$$

\#CAM-285/10. Received: 13/XI/10. Accepted: 25/IV/11. 
where $F$ and $G$ are given smooth functions, $D^{\alpha}$ is a fractional differential operator of order $\alpha$ in the Caputo's sense, $B_{j} ; j=0,1, \ldots, m-1$, are $m$ supplementary conditions and $u(x)$ is a solution to be determined.

Differential and integral equations involving derivatives of non-integer order have shown to be adequate models for various phenomena arising in damping laws, diffusion processes, models of earthquake [1], fluid-dynamics traffic model [2], mathematical physics and engineering [3], fluid and continuum mechanics [4], chemistry, acoustics and psychology [5].

Some numerical methods for the solution of FIDEs are presented in the literature. We can point out to the collocation method [6], Adomian decomposition method (ADM) [7]-[9], Spline collocation method [10], fractional differential transform method [11] and the method of combination of forward and central differences [12]. Out of the aforesaid methods, we desire to consider OTM for solving FIDEs.

Spectral methods provide a computational approach which achieved substantial popularity in the last three decades. Tau method is one of the most important spectral methods which is extensively applied for numerical solution of many problems. This method was invented by Lanczos [13] for solving ordinary differential equations (ODEs) and then the expansion of the method were done for many different problems such as partial differential equations (PDEs) [14]-[16], integral equations (IEs) [17], integro-differential equations (IDEs) [18] and etc. [19]-[22].

In this work, we are interested in solving FIDEs with an operational approach of the Tau method. Because in the Tau method, we are dealing with a system of equations wherein the matrix of unknown coefficients is sparse and can be easily invertible. Also, the differential and integral parts appearing in the equation is replaced by its operational Tau representation. Then, we obtain a system of algebraic equations wherein its solution is easy.

This work has been organized as follows. In Section 2, we briefly state the basic definitions of the fractional calculus. Section 3 is devoted to introduce the OTM and its application on FIDEs. In Section 4, the Legendre and Laguerre polynomials as base functions are considered. An efficient error estimation of the proposed method is presented in Section 5. The accuracy and efficiency of the scheme is investigated with three illustrative numerical experiments in Section 6. Finally, Section 7 consists of some obtained conclusions. 


\section{Basic definitions of the fractional calculus}

In this section, we give some basic definitions and properties of the fractional calculus theory, which are used in this work [3, 23].

Definition 1. A real function $u(x), x>0$ is said to be in the space $C_{\mu}, \mu \in \mathbb{R}$, if there exists a real number $p>\mu$, such that $u(x)=x^{p} v(x)$, where $v(x) \in$ $C[0, \infty)$ and it is said to be in the space $C_{\mu}^{m}$ iff $u^{(m)}(x) \in C_{\mu}, m \in \mathbb{N}$.

Definition 2. The Riemann-Liouville fractional integral operator of order $\alpha \geq 0$, of a function $u(x) \in C_{\mu}, \mu \geq-1$, is defined as:

$$
\begin{aligned}
& J^{\alpha} u(x)=\frac{1}{\Gamma(\alpha)} \int_{0}^{x}(x-t)^{\alpha-1} u(t) d t, \quad \alpha>0, x>0, \\
& J^{0} u(x)=u(x),
\end{aligned}
$$

where $\Gamma$ is the Gamma function. Some of the most important properties of operator $J^{\alpha}$ for $u(x) \in C_{\mu}, \mu \geq-1, \alpha, \beta \geq 0$ and $\gamma>-1$, are as follows:

i) $J^{\alpha} J^{\beta} u(x)=J^{\alpha+\beta} u(x)$,

ii) $J^{\alpha} J^{\beta} u(x)=J^{\beta} J^{\alpha} u(x)$,

iii) $J^{\alpha} x^{\gamma}=\frac{\Gamma(\gamma+1)}{\Gamma(\alpha+\gamma+1)} x^{\alpha+\gamma}$.

Definition 3. The fractional derivative of $u(x)$ in the Caputo's sense is defined as:

$$
D^{\alpha} u(x)=J^{m-\alpha} D^{m} u(x)=\frac{1}{\Gamma(m-\alpha)} \int_{0}^{x}(x-t)^{m-\alpha-1} u^{(m)}(t) d t,
$$

when $m-1<\alpha \leq m, m \in \mathbb{N}, x>0, u(x) \in C_{-1}^{m}$.

\section{Operational Tau method}

In this section, we state the structure of OTM and its application on FIDEs. Also, we present some preliminaries and notations using in this work. 
For any integrable functions $\psi(x)$ and $\phi(x)$ on $[a, b]$, we define the scalar product $\langle$,$\rangle by$

$$
\langle\psi(x), \phi(x)\rangle_{w}=\int_{a}^{b} \psi(x) \phi(x) \omega(x) d x,
$$

where $\|\psi\|_{w}^{2}=\langle\psi(x), \psi(x)\rangle_{\omega}$ and $\omega(x)$ is a weight function. Let $L_{\omega}^{2}[a, b]$ be the space of all functions $f:[a, b] \rightarrow \mathbb{R}$, with $\|f\|_{\omega}^{2}<\infty$.

The main idea of the method is to seek a polynomial to approximate $u(x) \in$ $L_{\omega}^{2}[a, b]$. Let $\phi_{x}=\left\{\phi_{i}(x)\right\}_{i=0}^{\infty}=\mathbf{\Phi} \mathbf{X}_{x}$ be a set of arbitrary orthogonal polynomial bases defined by a lower triangular matrix $\boldsymbol{\Phi}$ and $\mathbf{X}_{x}=\left[1, x, x^{2}, \ldots\right]^{T}$.

Lemma 1. Suppose that $u(x)$ is a polynomial as $u(x)=\sum_{i=0}^{\infty} u_{i} x^{i}=\mathbf{u} \mathbf{X}_{x}$, then we have:

$$
\begin{gathered}
D^{r}(x)=\frac{d^{r}}{d x^{r}} u(x)=\mathbf{u} \mathbf{M}^{r} \mathbf{X}_{x}, \quad r=0,1,2, \ldots, \\
x^{s} u(x)=\mathbf{u N}^{s} \mathbf{X}_{x}, \quad s=0,1,2, \ldots,
\end{gathered}
$$

and

$$
\int_{a}^{x} u(t) d t=\mathbf{u} \mathbf{P X} \mathbf{X}_{x}-\mathbf{u} \mathbf{P X} \mathbf{X}_{a}
$$

where $\mathbf{u}=\left[u_{0}, u_{1}, \ldots, u_{n}, \ldots\right], \mathbf{X}_{a}=\left[1, a, a^{2}, \ldots\right]^{T}, a \in \mathbb{R}$ and $\mathbf{M}, \mathbf{N}$ and $\mathbf{P}$ are infinite matrices with only nonzero elements $\mathbf{M}_{i+1, i}=i+1, \mathbf{N}_{i, i+1}=1$, $\mathbf{P}_{i, i+1}=\frac{1}{i+1}, i=0,1,2, \ldots$.

Proof. See [24].

Let us consider

$$
u(x)=\sum_{i=0}^{\infty} u_{i} \phi_{i}(x)=\mathbf{u} \boldsymbol{\Phi} \mathbf{X}_{x},
$$

to be an orthogonal series expansion of the exact solution of equations (1) and (2), where $\mathbf{u}=\left\{u_{i}\right\}_{i=0}^{\infty}$ is a vector of unknown coefficients, $\boldsymbol{\Phi} \mathbf{X}_{x}$ is an orthogonal basis for polynomials in $\mathbb{R}$.

In the Tau method, the aim is to convert equations (1) and (2) to an algebraic system using some operational matrices. First of all, we intend to describe an operational form of $D^{\alpha} u(x)$. In order to do this, we assert the process as follows. 
Using equations (3), (4) and (7); we get:

$$
D^{\alpha} u(x)=J^{m-\alpha} D^{m}\left(\mathbf{u} \boldsymbol{\Phi} \mathbf{X}_{x}\right)=J^{m-\alpha}\left(\mathbf{u} \boldsymbol{\Phi} \mathbf{M}^{m} \mathbf{X}_{x}\right)=\mathbf{u} \boldsymbol{\Phi} \mathbf{M}^{m} J^{m-\alpha}\left(\mathbf{X}_{x}\right) .
$$

Using property iii) of Definition 2, we have:

$$
\begin{gathered}
J^{m-\alpha}\left(\mathbf{X}_{x}\right)=\left[J^{m-\alpha}(1), J^{m-\alpha}(x), \ldots, J^{m-\alpha}\left(x^{\gamma}\right), \ldots\right]^{T} \\
=\left[\frac{\Gamma(1) x^{m-\alpha}}{\Gamma(m-\alpha+1)}, \frac{\Gamma(2) x^{m-\alpha+1}}{\Gamma(m-\alpha+2)}, \ldots, \frac{\Gamma(\gamma+1) x^{m-\alpha+\gamma}}{\Gamma(m-\alpha+\gamma+1)}, \ldots\right]^{T}=\Gamma \Pi,
\end{gathered}
$$

where $\boldsymbol{\Gamma}$ is an infinite diagonal matrix with elements

$$
\boldsymbol{\Gamma}_{i, i}=\frac{\Gamma(i+1)}{\Gamma(m-\alpha+i+1)}, i=0,1, \ldots,
$$

and

$$
\boldsymbol{\Pi}=\left[x^{m-\alpha}, x^{m-\alpha+1}, \ldots, x^{m-\alpha+\gamma}, \ldots\right]^{T} .
$$

By approximating $x^{m-\alpha+\gamma}, \gamma=0,1, \ldots$; as follows:

$$
x^{m-\alpha+\gamma}=\sum_{i=0}^{\infty} a_{\gamma, i} \phi_{i}(x)=\mathbf{a}_{\gamma} \boldsymbol{\Phi} \mathbf{X}_{x}, \quad \mathbf{a}_{\gamma}=\left[a_{\gamma, 0}, a_{\gamma, 1}, a_{\gamma, 2}, \ldots\right],
$$

we obtain:

$$
\begin{gathered}
\boldsymbol{\Pi}=\left[\mathbf{a}_{0} \boldsymbol{\Phi} \mathbf{X}_{x}, \mathbf{a}_{1} \boldsymbol{\Phi} \mathbf{X}_{x}, \ldots, \mathbf{a} \gamma \boldsymbol{\Phi} \mathbf{X}_{x}, \ldots\right]^{T}=\mathbf{A} \boldsymbol{\Phi} \mathbf{X}_{x}, \\
\mathbf{A}=\left[\mathbf{a}_{0}, \mathbf{a}_{1}, \ldots, \mathbf{a} \gamma, \ldots\right]^{T} .
\end{gathered}
$$

Substituting equation (10) in equation (9) and using equation (8); we obtain:

$$
D^{\alpha} u(x)=\mathbf{u} \boldsymbol{\Phi} \mathbf{M}^{m} \boldsymbol{\Gamma} \Pi=\mathbf{u} \boldsymbol{\Phi} \mathbf{M}^{m} \boldsymbol{\Gamma} \mathbf{A} \boldsymbol{\Phi} \mathbf{X}_{x}=\mathbf{u V} \boldsymbol{\Phi} \mathbf{X}_{x}, \quad \mathbf{V}=\boldsymbol{\Phi} \mathbf{M}^{m} \boldsymbol{\Gamma} \mathbf{A} .
$$

In next step, the aim is to linearize analytic functions $F(x, u(x))$ and $G(x$, $t, u(t))$. These functions can be written as:

$$
F(x, u(x))=\sum_{r=0}^{\infty} \sum_{p=0}^{\infty} f_{r p} x^{r} u^{p}(x),
$$

and

$$
G(x, t, u(t))=\sum_{r=0}^{\infty} \sum_{s=0}^{\infty} \sum_{p=0}^{\infty} g_{r s p} x^{r} t^{s} u^{p}(t) .
$$


Now, we state the following lemma and corollary.

Lemma 2. Let $\mathbf{X}_{x}=\left[1, x, x^{2}, \ldots\right]^{T}, \mathbf{u}=\left[u_{0}, u_{1}, u_{2}, \ldots\right]$ be infinite vectors and $\boldsymbol{\Phi}=\left[\phi_{0}\left|\phi_{1}\right| \phi_{2} \mid \ldots\right], \phi_{i}$ are infinite columns of matrix $\Phi$. Then, we have:

$$
\mathbf{X}_{x} \mathbf{u} \boldsymbol{\Phi} \mathbf{X}_{x}=\mathbf{U} \mathbf{X}_{x},
$$

where $\mathbf{U}$ is an upper triangular matrix as:

$$
\mathbf{U}_{i, j}=\left\{\begin{array}{cc}
\sum_{k=0}^{\infty} u_{k} \phi_{k, j-i}, & j \geq i, \quad i, j=0,1, \ldots, \\
0, & j<i, \quad i, j=0,1, \ldots
\end{array}\right.
$$

In addition, if we suppose that $u(x)=\mathbf{u} \boldsymbol{\Phi} \mathbf{X}_{x}$ represents a polynomial, then for any positive integer $p$, the relation

$$
u^{p}(x)=\mathbf{u} \boldsymbol{\Phi} \mathbf{U}^{p-1} \mathbf{X}_{x},
$$

is valid.

Proof. We have:

$$
\begin{aligned}
\mathbf{X}_{x} \mathbf{u} \boldsymbol{\Phi}= & {\left[1, x, x^{2}, \ldots\right]^{T}\left[\mathbf{u} \phi_{0}\left|\mathbf{u} \phi_{1}\right| \mathbf{u} \phi_{2} \mid \ldots\right] } \\
= & {\left[\begin{array}{llll}
\mathbf{u} \phi_{0} & \mathbf{u} \phi_{1} & \mathbf{u} \phi_{2} & \ldots \\
\mathbf{u} \phi_{0} x & \mathbf{u} \phi_{1} x & \mathbf{u} \phi_{2} x & \ldots \\
\mathbf{u} \phi_{0} x^{2} & \mathbf{u} \phi_{1} x^{2} & \mathbf{u} \phi_{2} x^{2} & \ldots \\
\vdots & \vdots & \vdots & \ddots
\end{array}\right], }
\end{aligned}
$$

therefore

$$
\begin{aligned}
\mathbf{X}_{x} \mathbf{u} \boldsymbol{\Phi} \mathbf{X}_{x}= & {\left[\begin{array}{llll}
\mathbf{u} \phi_{0} & \mathbf{u} \phi_{1} & \mathbf{u} \phi_{2} & \ldots \\
\mathbf{u} \phi_{0} x & \mathbf{u} \phi_{1} x & \mathbf{u} \phi_{2} x & \ldots \\
\mathbf{u} \phi_{0} x^{2} & \mathbf{u} \phi_{1} x^{2} & \mathbf{u} \phi_{2} x^{2} & \ldots \\
\vdots & \vdots & \vdots & \ddots
\end{array}\right]\left[1, x, x^{2}, \ldots\right]^{T} } \\
= & {\left[\begin{array}{c}
\sum_{i=0}^{\infty} \mathbf{u} \phi_{i} x^{i+0} \\
\sum_{i=0}^{\infty} \mathbf{u} \phi_{i} x^{i+1} \\
\sum_{i=0}^{\infty} \mathbf{u} \phi_{i} x^{i+2} \\
\vdots
\end{array}\right]=\left[\begin{array}{llll}
\mathbf{u} \phi_{0} & \mathbf{u} \phi_{1} & \mathbf{u} \phi_{2} & \cdots \\
0 & \mathbf{u} \phi_{0} & \mathbf{u} \phi_{1} & \cdots \\
0 & 0 & \mathbf{u} \phi_{0} & \cdots \\
\vdots & \vdots & \vdots & \ddots
\end{array}\right]\left[\begin{array}{c}
1 \\
x \\
x^{2} \\
\vdots
\end{array}\right], }
\end{aligned}
$$


if we call the last upper triangular coefficient matrix as $\mathbf{U}$, then we have:

$$
\begin{aligned}
\mathbf{U}_{i, j} & =\left\{\begin{array}{cc}
\mathbf{u} \phi_{j-i}, & j \geq i, \quad i, j=0,1, \ldots, \\
0, & j<i, \quad i, j=0,1, \ldots,
\end{array}\right. \\
& =\left\{\begin{array}{cc}
\sum_{k=0}^{\infty} u_{k} \phi_{k, j-i}, & j \geq i, \quad i, j=0,1, \ldots, \\
0, & j<i, \quad i, j=0,1, \ldots
\end{array}\right.
\end{aligned}
$$

Now, in order to prove equation (16), we apply induction. For $p=1$, it is obvious that $u(x)=\mathbf{u} \boldsymbol{\Phi} \mathbf{X}_{x}$. For $p=2$, we rewrite $u^{2}(x)=\mathbf{u} \boldsymbol{\Phi} \mathbf{X}_{x} \mathbf{u} \boldsymbol{\Phi} \mathbf{X}_{x}=$ $\mathbf{u} \boldsymbol{\Phi}\left(\mathbf{X}_{x} \mathbf{u} \boldsymbol{\Phi} \mathbf{X}_{x}\right)$ and using equation (14), we have:

$$
u^{2}(x)=\mathbf{u} \boldsymbol{\Phi} \mathbf{U} \mathbf{X}_{x},
$$

therefore, equation (16) is hold for $p=2$. Now, suppose that equation (16) is hold for $p=k$, then we must prove that the relation is valid for $s=k+1$. Thus,

$$
\begin{aligned}
u^{k+1}(x) & =u^{k}(x) u(x)=\left(\mathbf{u} \boldsymbol{\Phi} \mathbf{U}^{k-1} \mathbf{X}_{x}\right)\left(\mathbf{u} \boldsymbol{\Phi} \mathbf{X}_{x}\right) \\
& =\mathbf{u} \boldsymbol{\Phi} \mathbf{U}^{k-1}\left(\mathbf{X}_{x} \mathbf{u} \boldsymbol{\Phi} \mathbf{X}_{x}\right)=\mathbf{u} \boldsymbol{\Phi} \mathbf{U}^{k} \mathbf{X}_{x}
\end{aligned}
$$

So, equation (16) is proved.

We rewrite equation (12) as:

$$
F(x, u(x))=\sum_{r=0}^{\infty} \sum_{p=0}^{\infty} f_{r p} x^{r} u^{p}(x)=\sum_{r=0}^{\infty} f_{r 0} x^{r}+\sum_{r=0}^{\infty} \sum_{p=1}^{\infty} f_{r p} x^{r} u^{p}(x),
$$

The first summation can be considered as:

$$
\sum_{r=0}^{\infty} f_{r 0} x^{r}=\mathbf{f} \mathbf{X}_{x}, \quad \mathbf{f}=\left[f_{00}, f_{10}, f_{20}, \ldots\right] .
$$

For the second summation, using equations (5) and (16) yields:

$$
\begin{gathered}
\sum_{r=0}^{\infty} \sum_{p=1}^{\infty} f_{r p} x^{r} u^{p}(x)=\sum_{r=0}^{\infty} \sum_{p=1}^{\infty} f_{r p} x^{r} \mathbf{u} \boldsymbol{\Phi} \mathbf{U}^{p-1} \mathbf{X}_{x}=\mathbf{u} \boldsymbol{\Phi} \sum_{r=0}^{\infty} \sum_{p=1}^{\infty} f_{r p} x^{r} \mathbf{U}^{p-1} \mathbf{X}_{x} \\
=\mathbf{u} \boldsymbol{\Phi} \sum_{r=0}^{\infty} \sum_{p=1}^{\infty} f_{r p} \mathbf{U}^{p-1} \mathbf{N}^{r} \mathbf{X}_{x}=\mathbf{u} \boldsymbol{\Phi} \mathbf{F} \mathbf{X}_{x}, \quad \mathbf{F}=\left(\sum_{r=0}^{\infty} \sum_{p=1}^{\infty} f_{r p} \mathbf{U}^{p-1} \mathbf{N}^{r}\right)
\end{gathered}
$$


Therefore, we have:

$$
F(x, u(x))=\mathbf{f} \mathbf{X}_{x}+\mathbf{u} \boldsymbol{\Phi} \mathbf{F} \mathbf{X}_{x}=\left(\mathbf{f} \boldsymbol{\Phi}^{-1}+\mathbf{u} \boldsymbol{\Phi} \mathbf{F} \Phi^{-1}\right) \boldsymbol{\Phi} \mathbf{X}_{x} .
$$

In the same manner, we rewrite:

$$
\begin{aligned}
G(x, t, u(t)) & =\sum_{r=0}^{\infty} \sum_{s=0}^{\infty} \sum_{p=0}^{\infty} g_{r s p} x^{r} t^{s} u^{p}(t) \\
& =\sum_{r=0}^{\infty} \sum_{s=0}^{\infty} g_{r s 0} x^{r} t^{s}+\sum_{r=0}^{\infty} \sum_{s=0}^{\infty} \sum_{p=1}^{\infty} g_{r s p} x^{r} t^{s} u^{p}(t) .
\end{aligned}
$$

Using equation (5), the second summation is as:

$$
\begin{gathered}
\sum_{r=0}^{\infty} \sum_{s=0}^{\infty} \sum_{p=1}^{\infty} g_{r s p} x^{r} t^{s} u^{p}(t)=\sum_{r=0}^{\infty} \sum_{s=0}^{\infty} \sum_{p=1}^{\infty} g_{r s p} x^{r} t^{s} \mathbf{u} \Phi \mathbf{U}^{p-1} \mathbf{X}_{t} \\
=\mathbf{u} \boldsymbol{\Phi} \sum_{r=0}^{\infty} \sum_{s=0}^{\infty} \sum_{p=1}^{\infty} g_{r s p} x^{r} t^{s} \mathbf{U}^{p-1} \mathbf{X}_{t}=\mathbf{u} \boldsymbol{\Phi} \sum_{r=0}^{\infty} \sum_{s=0}^{\infty} \sum_{p=1}^{\infty} g_{r s p} x^{r} \mathbf{U}^{p-1} \mathbf{N}^{s} \mathbf{X}_{t} .
\end{gathered}
$$

Therefore,

$$
\begin{aligned}
\int_{0}^{x} G(x, t, u(t)) d t= & \sum_{r=0}^{\infty} \sum_{s=0}^{\infty} g_{r s 0} x^{r} \int_{0}^{x} t^{s} d t \\
& +\mathbf{u} \boldsymbol{\Phi} \sum_{r=0}^{\infty} \sum_{s=0}^{\infty} \sum_{p=1}^{\infty} g_{r s p} x^{r} \mathbf{U}^{p-1} \mathbf{N}^{s} \int_{0}^{x} \mathbf{X}_{t} d t .
\end{aligned}
$$

The first part easily can be written as follows:

$$
\sum_{r=0}^{\infty} \sum_{s=0}^{\infty} g_{r s 0} x^{r} \int_{0}^{x} t^{s} d t=\sum_{r=0}^{\infty} \sum_{s=0}^{\infty} g_{r s 0} \frac{x^{r+s+1}}{s+1}=\mathbf{g X},
$$

where

$$
[\mathbf{g}]_{i}=\left\{\begin{array}{cc}
0, & i=0 \\
\sum_{j=0}^{i-1} \frac{1}{i-j} g_{j(i-1-j) 0}, & i \geq 1
\end{array}\right.
$$


Using equations (5) and (6), the second part of equation (22) is written as follows:

$$
\begin{gathered}
\mathbf{u} \boldsymbol{\Phi} \sum_{r=0}^{\infty} \sum_{s=0}^{\infty} \sum_{p=1}^{\infty} g_{r s p} x^{r} \mathbf{U}^{p-1} \mathbf{N}^{s} \mathbf{P} \mathbf{X}_{x}=\mathbf{u} \boldsymbol{\Phi} \sum_{r=0}^{\infty} \sum_{s=0}^{\infty} \sum_{p=1}^{\infty} g_{r s p} \mathbf{U}^{p-1} \mathbf{N}^{s} \mathbf{P} \mathbf{M}^{r} \mathbf{X}_{x} \\
=\mathbf{u} \boldsymbol{\Phi} \mathbf{G} \mathbf{X}_{x}, \quad \mathbf{G}=\left(\sum_{r=0}^{\infty} \sum_{s=0}^{\infty} \sum_{p=1}^{\infty} g_{r s p} \mathbf{U}^{p-1} \mathbf{N}^{s} \mathbf{P} \mathbf{M}^{r}\right)
\end{gathered}
$$

Thus, we have:

$$
\int_{0}^{x} G(x, t, u(t)) d t=(\mathbf{g}+\mathbf{u} \boldsymbol{\Phi} \mathbf{G}) \mathbf{X}_{x}=\left(\mathbf{g} \boldsymbol{\Phi}^{-1}+\mathbf{u} \boldsymbol{\Phi} \mathbf{G} \Phi^{-1}\right) \boldsymbol{\Phi} \mathbf{X}_{x} .
$$

In addition, suppose that the supplementary conditions are generally as:

$$
B_{j} u(x)=\sum_{i=0}^{l} \sum_{k=0}^{q} b_{j i k} u^{(i)}\left(x_{k}\right)=\lambda_{j}, \quad 0 \leq x_{k} \leq x, j=0, \ldots, m-1 .
$$

Therefore, using equation (4) we have:

$$
\begin{aligned}
& B_{j} u(x)=\sum_{i=0}^{l} \sum_{k=0}^{q} b_{j i k} \mathbf{u} \boldsymbol{\Phi} \mathbf{M}^{i} \mathbf{X}_{x_{k}}=\mathbf{u b} \mathbf{b}_{j}=\lambda_{j}, \\
& \mathbf{b}_{j}=\sum_{i=0}^{l} \sum_{k=0}^{q} b_{j i k} \boldsymbol{\Phi} \mathbf{M}^{i} \mathbf{X}_{x_{k}} .
\end{aligned}
$$

Using equations (11), (18) and (27) we replace equation (1) by the following operational form:

$$
\mathbf{u V} \boldsymbol{\Phi} \mathbf{X}_{x}=\left(\mathbf{f} \Phi^{-1}+\mathbf{u} \boldsymbol{\Phi} \mathbf{F} \Phi^{-1}+\mathbf{g} \boldsymbol{\Phi}^{-1}+\mathbf{u} \boldsymbol{\Phi} \mathbf{G} \boldsymbol{\Phi}^{-1}\right) \boldsymbol{\Phi} \mathbf{X}_{x} .
$$

So, the residual $R(x)$ of equation (1), can be written as:

$$
R(x)=\left[(\mathbf{f}+\mathbf{g}) \boldsymbol{\Phi}^{-1}+\mathbf{u}\left(\Phi(\mathbf{F}+\mathbf{G}) \boldsymbol{\Phi}^{-1}-\mathbf{V}\right)\right] \boldsymbol{\Phi} \mathbf{X}_{x}=\mathbf{R} \boldsymbol{\Phi} \mathbf{X}_{x},
$$

where

$$
\mathbf{R}=\left[(\mathbf{f}+\mathbf{g}) \boldsymbol{\Phi}^{-1}+\mathbf{u}\left(\boldsymbol{\Phi}(\mathbf{F}+\mathbf{G}) \boldsymbol{\Phi}^{-1}-\mathbf{V}\right)\right]
$$


Now, we set the residual matrix $\mathbf{R}=0$ or we use the following inner products,

$$
\left\langle R(x), \phi_{k}(x)\right\rangle_{w}=0, \quad k=0,1, \ldots
$$

Therefore an infinite system of algebraic equations is obtained. Since, somewhere we require finite terms of approximation, then we must truncate the series to finite number of terms. Thus, we choose $n+1-m$ of the first equations in $\mathbf{R}$ and the following system is obtained:

$$
\begin{aligned}
\mathbf{R}_{i} & =0, & i & =0,1, \ldots, n-m, \\
\mathbf{u b}_{j} & =\lambda_{j}, & j & =0, \ldots, m-1,
\end{aligned}
$$

or

$$
\begin{array}{rlrl}
\left\langle R(x), \phi_{k}(x)\right\rangle_{w} & =0, & k & =0,1, \ldots, n-m, \\
\mathbf{u b}_{j}=\lambda_{j}, & j & =0, \ldots, m-1 .
\end{array}
$$

Solving the aforesaid system yields the unknown vector $\mathbf{u}=\left[u_{0}, u_{1}, \ldots, u_{n}\right]$. This is the so-called operational Tau method which is applicable for finite, infinite, regular and irregular domains.

We summarize OTM in the following algorithm.

\section{Algorithm of the method:}

Step 1: Choose suitable orthogonal bases $\phi(x)=\left\{\phi_{i}(x)\right\}_{i=0}^{n}$ and find nonsingular coefficient matrix $\boldsymbol{\Phi}$ and the compute $\boldsymbol{\Phi}^{-1}$.

Step 2: Consider $u(x)=\mathbf{u} \boldsymbol{\Phi} \mathbf{X}_{x}$ as the series expansion of exact solution.

Step 3: Use equations (4) through (11) to evaluate matrix $\mathbf{V}$.

Step 4: Use equations (18) and (27) to obtain vectors $f, g$ and matrices $F, G$.

Step 5: Compute matrix $\mathbf{R}$ from residual term $R(x, t)$ and set $\mathbf{R}=0$.

Step 6: Impose $m$ supplementary conditions $\mathbf{u b}_{j}=\lambda_{j}$ and solve the obtained system.

\section{Some orthogonal polynomials}

Orthogonal functions have received considerable attention in dealing with several problems. Their most important characteristic is reducing the computations and converting the problem to a system of algebraic equations. In this work, we use Legendre polynomials for finite domain $[0, h]$ and Laguerre polynomials for infinite domain $[0, \infty]$. 


\subsection{Legendre polynomials}

The well-known Legendre polynomials are defined as follows:

$$
\begin{gathered}
P_{0}(x)=1, P_{1}(x)=x, \quad x \in[-1,1], \\
P_{i}(x)=\left(2-\frac{1}{i}\right) x P_{i-1}(x)-\left(1-\frac{1}{i}\right) P_{i-2}(x), i \geq 2 .
\end{gathered}
$$

They are orthogonal on the interval $[-1,1]$ with respect to the weight function $w(x)=1$ and satisfy:

$$
\int_{-1}^{1} w(x) P_{m}(x) P_{n}(x) d x=\left\{\begin{array}{cc}
\frac{2}{2 n+1}, & m=n, \\
0, & m \neq n .
\end{array}\right.
$$

Since the interval of orthogonality of these polynomials may differ with the domain of the problem, we must shift the polynomial to the desired interval. Thus, to construct the shifted Legendre polynomials on arbitrary interval $[0, h]$, it is sufficient to do the change of variable: $x \rightarrow \frac{2 x-h}{h}$. So, the shifted Legendre polynomials are defined as follows:

$$
\begin{gathered}
P_{0}(x)=1, P_{1}(x)=\frac{2 x-h}{h}, x \in[0, h], \\
P_{i}(x)=\left(2-\frac{1}{i}\right)\left(\frac{2 x-h}{h}\right) P_{i-1}(x)-\left(1-\frac{1}{i}\right) P_{i-2}(x), i \geq 2,
\end{gathered}
$$

and satisfy:

$$
\int_{0}^{h} w(x) P_{m}(x) P_{n}(x) d x=\left\{\begin{array}{cc}
\frac{h}{2 n+1}, & m=n, \\
0, & m \neq n .
\end{array}\right.
$$

\subsection{Laguerre polynomials}

Laguerre polynomials are defined as follows:

$$
\begin{gathered}
L_{0}(x)=1, L_{1}(x)=1-x, \quad x \in[0, \infty), \\
L_{i}(x)=\left(\frac{2 i-1-x}{i}\right) L_{i-1}(x)-\left(\frac{i-1}{i}\right) L_{i-2}(x), i \geq 2 .
\end{gathered}
$$


They are orthogonal on the interval $[0, \infty)$ with respect to the weight function $w(x)=e^{-x}$ and satisfy:

$$
\int_{0}^{\infty} w(x) L_{m}(x) L_{n}(x) d x= \begin{cases}1, & m=n \\ 0, & m \neq n .\end{cases}
$$

\section{Error estimation}

In this section, an error estimation for the approximate solution of equation (1) with supplementary conditions (2) is obtained. Let us call $e_{n}(x)=u(x)-u_{n}(x)$ as the error function of the approximate solution $u_{n}(x)$ to $u(x)$ where $u(x)$ is the exact solution of equation (1). Hence, $u_{n}(x)$ satisfies the following equations:

$$
\begin{aligned}
D^{\alpha} u_{n}(x)= & F\left(x, u_{n}(x)\right)+\int_{0}^{x} G\left(x, t, u_{n}(t)\right) d t+H_{n}(x), \\
& m-1<\alpha \leq m, \\
B_{j} u_{n}(x)= & \lambda_{j}, \quad \lambda_{j} \in \mathbb{R}, \quad j=0,1,2, \ldots, m-1 .
\end{aligned}
$$

The perturbation term $H_{n}(x)$ can be obtained by substituting the computed solution $u_{n}(x)$ into the equation:

$$
H_{n}(x)=D^{\alpha} u_{n}(x)-F\left(x, u_{n}(x)\right)-\int_{0}^{x} G\left(x, t, u_{n}(t)\right) d t .
$$

We proceed to find an approximation $e_{n, N}(x)$ to the error function $e_{n}(x)$ in the same way as we did before for the solution of equation (1). Note that $N$ is the degree of approximation of $u_{n}(x)$. By subtracting equations (31) and (32) from equations (1) and (2), respectively we have:

$$
\begin{aligned}
& D^{\alpha}\left(u(x)-u_{n}(x)\right)=F\left(x, u(x)-u_{n}(x)\right) \\
& \quad+\int_{0}^{x} G\left(x, t, u(t)-u_{n}(t)\right) d t-H_{n}(x), \\
& B_{j}\left(u(x)-u_{n}(x)\right)=0,
\end{aligned}
$$

or

$$
\begin{aligned}
& D^{\alpha}\left(e_{n}(x)\right)=F\left(x, e_{n}(x)\right)+\int_{0}^{x} G\left(x, t, e_{n}(t)\right) d t-H_{n}(x), \\
& B_{j}\left(e_{n}(x)\right)=0 .
\end{aligned}
$$


It should be noted that in order to construct the approximate $e_{n, N}(x)$ to $e_{n}(x)$, only the related equations like as equations (7) through (31) needs to be recomputed and the structure of the method remains the same.

\section{Illustrative numerical experiments with some comments}

In this section, four experiments of linear and nonlinear FIDEs are given to illustrate the results. In all experiments, we consider the shifted Legendre polynomials as basis functions for finite domains and Laguerre polynomials for infinite domains. The computations associated with the experiments discussed above were performed in Maple 13 on a PC with a CPU of $2.4 \mathrm{GHz}$.

Experiment 6.1. Consider the following FIDE [6, 7]:

$$
D^{0.75} u(x)=-\left(\frac{x^{2} e^{x}}{5}\right) u(x)+\frac{x^{2.25}}{\Gamma(3.25)}+\int_{0}^{x} t e^{x} u(t) d t, \quad x>0,
$$

with the initial condition: $u(0)=0$

The exact solution is: $u(x)=x^{3}$.

We have solved this experiment using OTM with Laguerre polynomials and some approximations are obtained as follows:

$$
\begin{array}{lll}
n=0: & \mathbf{u}=[0], & u_{0}(x)=0, \\
n=1: & \mathbf{u}=[-1.536502,1.536502], & u_{1}(x)=-1.536502 x, \\
n=2: & \mathbf{u}=[-0.701605,1.413861,-0.712256], & u_{2}(x)=0.010651 x-0.356128 x^{2}, \\
n=3: & \mathbf{u}=[6,-18,18,-6], & u_{4}(x)=x^{3}, \\
n=4: & \mathbf{u}=[6,-18,18,-6,0], & u_{4}(x)=x^{3}, \\
n=5: & \mathbf{u}=[6,-18,18,-6,0,0], & u_{5}(x)=x^{3},
\end{array}
$$

and so on.

Thus, we have $u(x)=x^{3}$ which is the exact solution of the problem.

Experiment 6.2. Consider the following nonlinear FIDE [8]:

$$
D^{\alpha} u(x)=1+\int_{0}^{x} e^{-t} u^{2}(t) d t, \quad 0 \leq x \leq 1, \quad 3<\alpha \leq 4,
$$


with the boundary conditions:

$$
\begin{aligned}
& u(0)=u^{\prime}(0)=1, \\
& u(1)=u^{\prime}(1)=e .
\end{aligned}
$$

The only case which we know the exact solution for $\alpha=4$ is: $u(x)=e^{x}$.

We have solved this experiment for $n=7$ with different $\alpha$ and have compared it with the closed form series solutions of the exact solution obtained by ADM

\begin{tabular}{|c|c|c|c|c|c|c|}
\hline \multirow[b]{2}{*}{$x$} & \multicolumn{2}{|c|}{$\alpha=3.25$} & \multicolumn{2}{|c|}{$\alpha=3.5$} & \multicolumn{2}{|c|}{$\alpha=3.75$} \\
\hline & $u_{\mathrm{ADM}}$ & $u_{\mathrm{OTM}}$ & $u_{\mathrm{ADM}}$ & $u_{\mathrm{OTM}}$ & $u_{\mathrm{ADM}}$ & $u_{\mathrm{OTM}}$ \\
\hline 0.0 & 1.000000 & 1.000000 & 1.000000 & 1.000000 & 1.000000 & 1.000000 \\
\hline 0.1 & 1.106551 & 1.107575 & 1.106750 & 1.106693 & 1.106151 & 1.105930 \\
\hline 0.2 & 1.223931 & 1.225838 & 1.224323 & 1.224220 & 1.223227 & 1.222813 \\
\hline 0.3 & 1.353200 & 1.355724 & 1.353755 & 1.353600 & 1.352308 & 1.351741 \\
\hline 0.4 & 1.495600 & 1.498421 & 1.496270 & 1.496050 & 1.494635 & 1.493962 \\
\hline 0.5 & 1.652553 & 1.655348 & 1.653273 & 1.652982 & 1.651615 & 1.650890 \\
\hline 0.6 & 1.825654 & 1.828146 & 1.826354 & 1.826006 & 1.824823 & 1.824110 \\
\hline 0.7 & 2.016687 & 2.018671 & 2.017294 & 2.016931 & 2.016023 & 2.015390 \\
\hline 0.8 & 2.227634 & 2.228987 & 2.228084 & 2.227770 & 2.227176 & 2.226696 \\
\hline 0.9 & 2.460690 & 2.461367 & 2.460931 & 2.460744 & 2.460458 & 2.460196 \\
\hline 1.0 & 2.718281 & 2.718281 & 2.718281 & 2.718281 & 2.718281 & 2.718281 \\
\hline
\end{tabular}
[8]. The comparison is shown in Table 1.

Table 1 - Comparison of the solutions of ADM and OTM for different $\alpha$ of Experiment 6.2.

From the numerical results in Table 1, it is easy to conclude that obtained results by OTM are in good agreement with those obtained using the ADM. In this experiment, the exact solution is not known, so a main question arises that which method is more accurate. We conclude that OTM is more accurate by considering the following notation and discussion.

Note 1. In the theory of fractional calculus, it is obvious that when the fractional derivative $\alpha(m-1<\alpha \leq m)$ tends to positive integer number $m$, then the approximate solution continuously tends to the exact solution of the problem with derivation $m$.

A closer look at the values obtained by ADM in Table 1 do not have this characteristic. The values for $\alpha=3.25$ are less than the values for $\alpha=3.5$, 
so the values for $\alpha=3.5$ must be less than the values for $\alpha=3.75$. But this fact have not occurred for ADM solutions but for OTM solutions, we have the reduction in the results. Therefore OTM is more reliable than ADM.

Moreover, for $\alpha=4$, using Legendre polynomials, the following sequences of approximate solution is obtained:

$$
\begin{array}{ll}
n=4: \quad \mathbf{u}=\left[\frac{103}{60}, \frac{101}{120}, \frac{23}{168}, \frac{1}{80}, \frac{1}{1680}\right], \\
u_{4}(x)=1+x+\frac{x^{2}}{2}+\frac{x^{3}}{6}+\frac{x^{4}}{24}, \\
n=5: \quad \mathbf{u}=\left[\frac{1237}{720}, \frac{473}{560}, \frac{281}{2016}, \frac{59}{4320}, \frac{1}{1120}, \frac{1}{30240}\right], \\
n=6: \quad \mathbf{u}=\left[\frac{433}{252}, \frac{1893}{2240}, \frac{1691}{12096}, \frac{1}{72}, \frac{3}{3080}, \frac{1}{20160}, \frac{1}{665280}\right], \\
u_{5}(x)=1+x+\frac{x^{2}}{2}+\frac{x^{3}}{6}+\frac{x^{4}}{24}+\frac{x^{5}}{120}, \\
u_{6}(x)=1+x+\frac{x^{2}}{2}+\frac{x^{3}}{6}+\frac{x^{4}}{24}+\frac{x^{5}}{120}+\frac{x^{6}}{720},
\end{array}
$$

and so on.

Thus, we obtain:

$$
u_{n}(x)=1+x+\frac{x^{2}}{2 !}+\frac{x^{3}}{3 !}+\frac{x^{4}}{4 !}+\cdots+\frac{x^{n}}{n !} .
$$

This has the closed form $u(x)=e^{x}$, which is the exact solution of the problem. Thus, for positive integer derivatives, if the exact solution exists, then OTM produces its series solution.

Experiment 6.3. Consider the following nonlinear FIDE:

$$
\begin{aligned}
D^{\alpha} u(x)= & x^{3}\left(-1+e^{\sin x}\right)-\sin x-\int_{0}^{x} x^{3} \cos t e^{u(t)} d t \\
& x>0,1<\alpha \leq 2
\end{aligned}
$$


with the initial conditions:

$$
u(0)=0, u^{\prime}(0)=1 .
$$

The only case which we know the exact solution for $\alpha=2$ is $u(x)=\sin x$.

We have solved this experiment for $n=5$ with different $\alpha$ and have used Laguerre polynomials as basis functions. The results are given in Table 2.

\begin{tabular}{|c|ccccc|}
\hline$x$ & $\alpha=1.25$ & $\alpha=1.5$ & $\alpha=1.75$ & $\alpha=2$ & $u_{\text {Exact }}$ \\
\hline 0.0 & 0.000000 & 0.000000 & 0.000000 & 0.000000 & 0.000000 \\
0.1 & 0.097355 & 0.098677 & 0.099419 & 0.099833 & 0.099833 \\
0.2 & 0.188547 & 0.193716 & 0.196812 & 0.198669 & 0.198669 \\
0.3 & 0.272375 & 0.283807 & 0.290975 & 0.295520 & 0.295520 \\
0.4 & 0.347526 & 0.367799 & 0.380815 & 0.389418 & 0.389418 \\
0.5 & 0.412246 & 0.444601 & 0.465333 & 0.479427 & 0.479425 \\
0.6 & 0.464013 & 0.513081 & 0.543605 & 0.564648 & 0.564642 \\
0.7 & 0.499207 & 0.571968 & 0.614770 & 0.644233 & 0.644217 \\
0.8 & 0.512786 & 0.619749 & 0.678009 & 0.717397 & 0.717356 \\
0.9 & 0.497956 & 0.654568 & 0.732528 & 0.783420 & 0.783326 \\
1.0 & 0.445845 & 0.674130 & 0.777544 & 0.841666 & 0.841470 \\
\hline
\end{tabular}

Table 2 - OTM solutions for $n=5$ with different $\alpha$ of Experiment 6.3.

For $\alpha=2$, using Laguerre polynomials, the following sequence of approximate solution is obtained:

$$
\begin{array}{lll}
n=0: & \mathbf{u}=[0], & u_{0}(x)=0, \\
n=1: & \mathbf{u}=[1,-1], & u_{1}(x)=x, \\
n=2: & \mathbf{u}=[1,-1,0], & u_{2}(x)=x, \\
n=3: & \mathbf{u}=[0,2,-3,1], & u_{3}(x)=x-\frac{x^{3}}{6}, \\
n=4: & \mathbf{u}=[0,2,-3,1,0], & u_{4}(x)=x-\frac{x^{3}}{6}, \\
n=5: & \mathbf{u}=[1,-3,7,-9,5,-1], & u_{5}(x)=x-\frac{x^{3}}{6}+\frac{x^{5}}{120} \\
n=6: & \mathbf{u}=[1,-3,7,-9,5,-1,0], & u_{6}(x)=x-\frac{x^{3}}{6}+\frac{x^{5}}{120}
\end{array}
$$

and so on. 
Thus, we obtain:

$$
u_{2 n+1}(x)=x-\frac{x^{3}}{3 !}+\frac{x^{5}}{5 !}+\cdots+(-1)^{n} \frac{x^{2 n+1}}{(2 n+1) !} .
$$

This has the closed form $u(x)=\sin x$, which is the exact solution of the problem.

Experiment 6.4. Consider the following nonlinear FIDE:

$$
\begin{gathered}
D^{\alpha} u(x)=e^{-x}+\frac{2}{3}\left(e^{-\frac{3}{2} x}-1\right)+\int_{0}^{x} e^{-t} \sqrt{u(t)} d t, \\
0 \leq x \leq 1,1<\alpha \leq 2,
\end{gathered}
$$

with the initial conditions:

$$
u(0)=1, u^{\prime}(0)=-1
$$

The only case which we know the exact solution for $\alpha=2$ is: $u(x)=e^{-x}$.

We have solved this experiment for $n=4$ with different $\alpha$ and used shifted Legendre polynomials as basis functions. Figure 1 shows the approximate solutions and illustrates the aforesaid fact given by note 1 .

The following figure illustrates the convergency of the method and the fact that the method tends continuously to the exact solution if fractional derivations tend to an integer order.

For $\alpha=2$, using shifted Legendre polynomials, the following sequence of approximate solution is obtained:

$$
\begin{array}{lll}
n=0: & \mathbf{u}=[1], & u_{0}(x)=1, \\
n=1: \quad \mathbf{u}=\left[\frac{1}{2},-\frac{1}{2}\right], & u_{1}(x)=1-x, \\
n=2: \quad \mathbf{u}=\left[\frac{2}{3},-\frac{1}{12}, \frac{1}{2}\right], & u_{2}(x)=1-x+\frac{x^{2}}{2}, \\
n=3: & \mathbf{u}=\left[\frac{5}{8},-\frac{13}{40}, \frac{1}{24},-\frac{1}{120}\right], & u_{3}(x)=1-x+\frac{x^{2}}{2}-\frac{x^{3}}{6},
\end{array}
$$

and so on. 


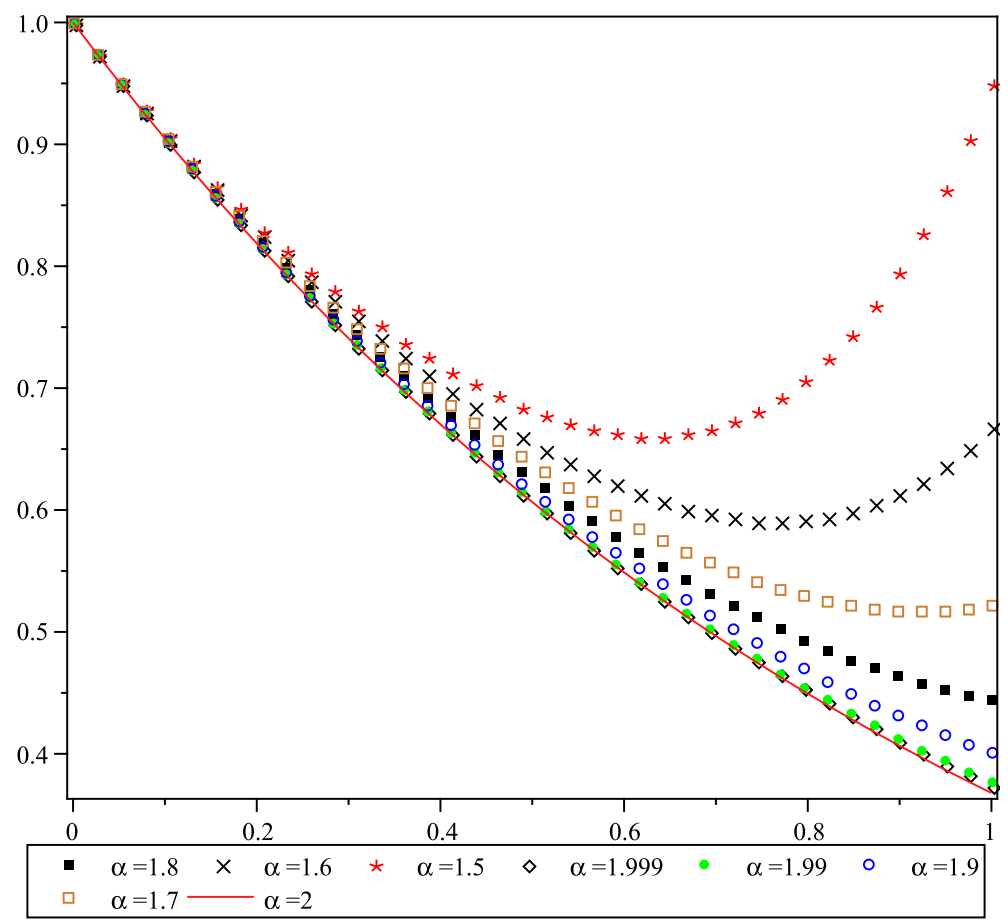

Figure 1 - The graphs of the approximate solutions for $n=4$ with different $\alpha$ of Experiment 6.4.

Thus, we obtain:

$$
u_{n}(x)=1-x+\frac{x^{2}}{2 !}-\frac{x^{3}}{3 !}+\cdots+(-1)^{n} \frac{x^{n}}{n !} .
$$

This has the closed form $u(x)=e^{-x}$, which is the exact solution of the problem.

\section{Conclusion}

In this work, operational Tau method is employed successfully to solve the FIDEs. Arbitrary orthogonal polynomial bases were applied as basis functions. Reducing the FIDEs to algebraic equations is the first characteristics of the proposed method. The applications of OTM on some problems including linear and nonlinear terms are considered and some useful results are obtained. 
The most important ones are the simplicity of the method, reducing the computations using orthogonal polynomials and having low run time of its algorithm. Furthermore, this method yields the desired accuracy only in a few terms in a series form of the exact solution. All of these advantages of the OTM to solve nonlinear problems assert the method as a convenient, reliable and powerful tool.

\section{REFERENCES}

[1] J.H. He, Nonlinear oscillation with fractional derivative and its applications. In: International Conference on Vibrating Engineering, Dalian, China (1998), 288-291.

[2] J.H. He, Some applications of nonlinear fractional differential equations and their approximations. Bull. Sci. Technol., 15 (1999), 86-90.

[3] I. Podlubny, Fractional Differential Equations. Academic Press, New York (1999).

[4] F. Mainardi, Fractals and Fractional Calculus Continuum Mechanics. Springer Verlag, (1997), 291-348.

[5] W.M. Ahmad and R. El-Khazali, Fractional-order dynamical models of love. Chaos, Solitons \& Fractals, 33 (2007), 1367-1375.

[6] E.A. Rawashdeh, Numerical solution of fractional integro-differential equations by collocation method. Applied Mathematics and Computation, 176 (2005), 1-6.

[7] R.C. Mittal and R. Nigam, Solution of fractional integro-differential equations by adomian decomposition method. Int. J. of Appl. Math. and Mech., 4 (2008), 87-94.

[8] S. Momani and M.A. Noor, Numerical methods for fourth-order fractional integro-differential equations. Applied Mathematics and Computation, 182 (2006), 754-760.

[9] W.G. El-Sayed and A.M.A. El-Sayed, On the functional integral equations of mixed type and integro-differential equations of fractional orders. Applied Mathematics and Computation, 154 (2004), 461-467.

[10] A. Pedas and E. Tamme, Spline collocation method for integro-differential equations with weakly singular kernels. Journal of Computational and Applied Mathematics, 197 (2006), 253-269.

[11] D. Nazari and S. Shahmorad, Application of the fractional differential transform method to fractional-order integro-differential equations with nonlocal boundary conditions. Journal of Computational and Applied Mathematics, 234 (2010), 883-891. 
[12] M.F. Al-Jamal and E.A. Rawashde, The Approximate Solution of Fractional Integro-Differential Equations. Int. J. Contemp. Math. Sciences, 4 (2009), 1067-1078.

[13] C. Lanczos, Trigonometric interpolation of empirical and analytical functions. J. Math. Phys., 17 (1938), 123-199.

[14] K.M. Liu and E.L. Ortiz, Numerical solution of eigenvalue problems for partial differential equations with the Tau-lines method. Comp. Math. Appl. B, 12 (1986), 1153-1168.

[15] E.L. Ortiz and K.S. Pun, Numerical solution of nonlinear partial differential equations with Tau method. J. Comp. Appl. Math., 12 (1985), 511-516.

[16] E.L. Ortiz and H. Samara, Numerical solution of partial differential equations with variable coefficients with an operational approach to the Tau method. Comp. Math. Appl., 10 (1984), 5-13.

[17] M.K. EL-Daou and H.G. Khajah, Iterated solutions of linear operator equations with the Tau method. Math. Comput., 66(217) (1997), 207-213.

[18] J. Pour-Mahmoud, M.Y. Rahimi-Ardabili and S. Shahmorad, Numerical solution of the system of Fredholm integro-differential equations by the Tau method. Applied Mathematics and Computation, 168 (2005), 465-478.

[19] K.M. Liu and E.L. Ortiz, Approximation of eigenvalues defined by ordinary differential equations with the Tau method. Matrix Pencils, Springer, Berlin, (1983), 90-102.

[20] K.M. Liu and E.L. Ortiz, Tau method approximation of differential eigenvalue problems where the spectral parameter enters nonlinearly. J. Comput. Phys., 72 (1987), 299-310.

[21] K.M. Liu and E.L. Ortiz, Numerical solution of ordinary and partial functionaldifferential eigenvalue problems with the Tau method. Computing, 41 (1989), 205-217.

[22] E.L. Ortiz and H. Samara, Numerical solution of differential eigenvalue problems with an operational approach to the Tau method. Computing, 31 (1983), 95-103.

[23] S. Samko, A. Kilbas and O. Marichev, Fractional Integrals and Derivatives. Gordon and Breach, Yverdon (1993).

[24] K.M. Liu and C.K. Pan, The automatic solution to systems of ordinary differential equations by the Tau method. Computers Math. Applicat., 38 (1999), 197-210. 